\title{
Modèle de crue dans un petit bassin urbain : premiers résultats d'une recherche expérimentale
}

\section{Runoff models in a small urban watershed: first results of an experimental study}

\author{
F. Callomino, C. Colosimo, V.A. Copertino et P. Veltri \\ Dipartimento di Difesa del Suolo, Università della Calabria, \\ Cosenza, Italie
}

\section{Introduction}

Ces dernières années, on a pu constater un intérêt croissant pour les problèmes relatifs à la formation des débits de crue dans les bassins urbains.

Les résultats de nombreuses enquêtes expérimentales et les travaux de quelques commissions d'études telle que :

- la "Commissione per lo studio delle portate di piena nelle fognature" [1] en Italie,

- 1" "Urban Water Resources Research Council" de l'American Society of Civil Engineers aux Etats-Unis, - la "Commission interministérielle Loriferne" en France,

ont stimulé de remarquables progrès dans le domaine des modèles mathématiques traduisant la transformation des précipitations en écoulements dans les bassins versants urbains.

Cependant, dans le calcul des égouts il y a de nombreux éléments d'incertitude. Ils dépendent d'une série de choix que le projeteur doit faire, souvent avec des éléments d'appréciation insuffisants quant à la pluie de projet, le modèle de simulation des phénomènes, les types de conduites ou d'ouvrages les plus appropriés au problème posé. Les investissements considérables consacrés à la création ou la restauration des égouts pluviaux font qu'une attention accrue et des crédits sont aujourd'hui accordés à des études visant à réduire le champ de ces incertitudes, et aboutissant notamment à des modèles de calcul des ouvrages fiables (adéquation des modèles) extrapolables et facilement adoptables par les "hommes de terrain" [2], [3].

Dans le but d'améliorer la connaissance des phénomènes hydrologiques urbains on a installé, en 1978, une station expérimentale pour la mesure des précipitations et des écoulements, dans l'agglomération urbaine de Luzzi, Cosenza, Italie. En employant les enregistrements de pluie et de débits, quelques modèles simples, du type pluie-ruissellement, particulièrement adaptés aux caractéristiques du bassin étudié, ont été appliqués.

\section{Modèles de transf ormation précipitations-écoule-} ments pour bassins urbains

A l'heure actuelle la pratique des projets d'égouts pluviaux suit des méthodes traditionnelles, basées sur des modèles de calcul simplifiés et sur des critères de choix des coefficients et d'intensité de pluie critique de fréquence prédéterminée. En outre, le calcul est souvent limité à la seule estimation du débit de pointe.

Abstraction faite des phénomènes physiques propres à la transformation précipitations-écoulements, on peut exprimer la relation entrée-sortie du système constitué par le bassin versant sous la forme tout à fait générale [4]

$$
Q=F I
$$

où $Q$ est la sortie, $I$ est l'entrée, $F$ une fonction de transfert

Dans ce schéma il est possible de placer la méthode rationnelle; en admettant comme entrée l'intensité de pluie moyenne $i\left(t_{c}\right)$ sur une durée que l'on pose, en général, égale au temps de concentration $t_{c}$, et comme opérateur de transformation le produit du coefficient $C$ par la surface $A$ du bassin versant; la relation (1) devient

$$
Q_{p}=C A i\left(t_{c}\right)
$$

où $Q_{p}$ est le débit de pointe.

Dans le modèle représenté par la relation (2), le coefficient $C$ tient compte de tous les phénomènes liés à l'infiltration, et à l'évaporation, etc.

Les défauts du modèle résident soit dans le fait que l'on considère $t$ et $C$ comme des valeurs constantes pour un point du bassin, soit dans l'indétermination de la valeur de $t_{c}[5]$. En outre, la méthode rationnelle bien que s'appuyant sur un support expérimental important [6], ne tient pas compte exactement de l'effet capacitif du bassin et du réseau. 
La méthode qu'on attribue à $\mathrm{M}$. Caquot n'est en réalité qu'une modification de la méthode rationnelle, dans laquelle on tient compte aussi de l'effet de capacité du bassin [7].

Avec les méthodes complètes, la relation (1) conduit à la connaissance de l'hydrogramme de ruissellement $Q(t)$ à partir de celle du hyétogramme de pluie efficace $I(t)$.

Dans la méthode volumétrique, la relation (1) prend la forme largement connue

$$
I(t)-Q(t)=\frac{d S(t)}{d t}
$$

où $d S(t)$ est le stockage pendant le temps $d t$, dans le système.

Dans l'école hydraulique italienne, on assigne un volume constant à la capacité superficielle et on suppose l'écoulement dans chaque canal comme étant constitué d'une succession chronologique d'écoulements uniformes. Le lien entre le débit sortant et le volume stocké étant ainsi connu, une fois fixée la loi intensitédurée, on parvient à la détermination de la durée critique qui donne le maximum $Q_{p}$ et, par intégration de la relation (3) sur cette durée critique, on calcule la valeur du même $Q_{p}$.

Dans le cas de réseaux de canaux, l'équation (3) peut être intégrée successivement pour chacun d'eux, étant donné qu'on connait l'afflux $I(t)$. Dans le cas particulier où $Q(t)$ et $S(t)$ sont liés par la relation du réservoir linéaire

$$
S(t)=k Q(t)
$$

où $k$ est une constante ayant la dimension d'un temps, et si le canal est vide au début de la pluie, l'intégrale de la relation (3) devient :

$$
Q(t)=\frac{1}{k} \int_{0}^{t} e^{-(t-\tau) / k} I(\tau) d \tau
$$

En assimilant le bassin à un canal prismatique de longueur $L$, parcouru par un courant en écoulement uniforme, de section mouillée $\Omega(t)$, la relation (4) conduit à l'équation :

$$
L \Omega(t)=k \Omega(t) v(t)
$$

où $v(t)$ est la vitesse moyenne dans le canal; le paramètre du modèle devient donc

$$
k=\frac{L}{v(t)}
$$

En séparant l'effet de la capacité du bassin de celui des canaux, et en supposant avoir des éléments linéaires distincts et autonomes placés en série, il est possible de reconstruire l'hydrogramme $Q(t)$ par l'évaluation de chaque valeur de $k[8]$. Dans d'autres recherches, les phénomènes superficiels dans le bassin et ceux de son réseau élémentaire de drainage sont représentés par un réservoir linéaire, tandis que la propagation des hydrogrammes dans le réseau des collecteurs est obtenue par l'équation de Muskingum :

$$
S(t)=K[x I(t)+(1-x) Q(t)]
$$

dans laquelle $K$ est encore une constante ayant la dimension d'un temps et $x$ un coefficient de pondération entre les débits entrants et sortants [9].

Les processus complexes de transformation de la pluie en ruissellement ou écoulement peuvent sans doute être décrits par l'hydraulique des régimes tran- sitoires. L'identification des opérateurs de transformation serait malgré tout très délicate en raison de la grande hétérogénéité des surfaces d'écoulement et de la variabilité dans le temps de leurs caractéristiques hydrauliques.

Ces modèles, dits à simulation détaillée, même s'ils ont été récemment employés avec un certain succès dans les bassins urbains [10], [11], [12], semblent souffrir de difficultés sérieuses dans le calcul et dans lacquisition des nombreuses informations hydrologiques et morphologiques nécessaires à l'identification des paramètres des équations.

Il semble donc inutile d'employer des modèles très sophistiqués de la composante hydraulique tandis que la modelisation de la composante hydrologique souffre d'importantes approximations [8]. Une récente étude théorique [13] a d'ailleurs montré que l'intégration de l'équation différentielle employée dans la méthode italienne, pour obtenir la propagation de la crue dans un réseau d'égouts urbains, donne des résultats qui s'écartent peu de ceux obtenus par la solution des équations complètes de De Saint-Venant.

Même les modèles à simulation détaillée, comme l'observe Dooge [14], [15], peuvent être considérés comme des modèles conceptuels. En effet, les solutions approximatives fournies pour l'écoulement superficiel et pour l'écoulement dans la canalisation conduisent à des relations assez simples entre le débit et le volume emmagasiné, ou entre le débit de pluie et celui qui s'écoule à l'exutoire, tels, cependant, qu'elles peuvent schématiser le bassin comme un ensemble d'éléments conceptuels.

D'une manière analogue un modèle qui emploie la convolution peut être considéré comme un modèle conceptuel si le noyau de l'intégrale est propre à un élément conceptuel (réservoir linéaire, canal linéaire, etc.).

Un tel modèle, dérivé de l'analyse des systèmes, ignore la complexité des processus hydrologiques et permet de traiter globalement la relation précipitationsécoulements.

Cependant il est évident, lorsque le modèle n'est pas linéaire, qu'il n'est plus possible d'obtenir l'hydrogramme résultant d'u n hyétogramme, par simple convolution de ce dernier avec la réponse impulsionnelle (hydrogramme unitaire instantané, IUH selon la terminologie anglaise). Donc, les tentatives pour simuler le système par de simples éléments conceptuels nonlinéaires, comme le réservoir non-linéaire ou le modèle de Muskingum non-linéaire, apparaissent difficiles.

En gardant la nature linéaire d'un modèle on peut rechercher par des techniques d'optimisation les "meilleures" valeurs numériques de ses paramètres "satisfaisants" à l'observation. Les valeurs des paramètres de l'IUH peuvent être ensuite "expliquées", à l'aide de techniques d'analyse multivariable, par celles des caractéristiques de la pluie ou des bassins [16], [17].

On peut alors avoir espoir d'extrapoler les modèles hors de leur domaine de vérification expérimentale. Pour avancer dans cette voie il convient d'équiper de stations de mesure de nombreux bassins de caractéristiques différentes. 


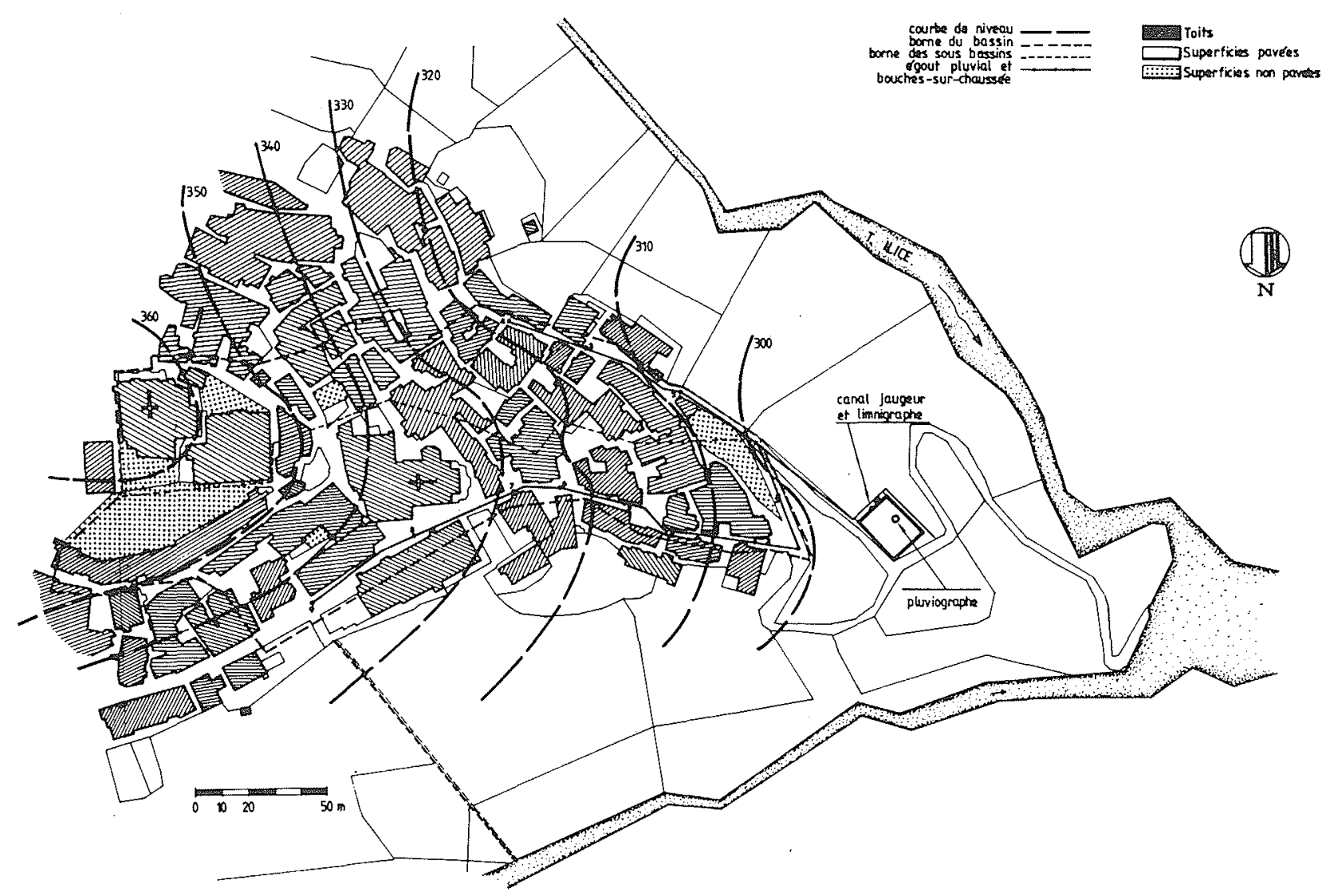

Figure 1 - Bassin urbain expérimental, Luzzi, Cosenza, Italie

\section{Description du bassin expérimental de Luzzi}

Le bassin expérimental occupe une partie de l'agglomération de Luzzi, pour une surface de 1,734 ha (fig.1). La structure urbaine, qui est typique de beaucoup de villages de montagne des Apennins, présente un réseau routier constitué par un enchevêtrement de nuelles et d'escaliers, très étroits et raides, avec des pentes qui varient entre $5 \%$ et $35 \%$. La chaussée, généralement pavée ou en béton, présente d'innombrables crevasses et fentes, et de fréquents obstacles représentés par les rampes d'accès aux immeubles. Pour une grande partie, la surface du bassin est représentée par des toits, tandis que seulement $15 \%$ est constitué par des potagers ou par des surfaces qui n'ont, de toute manière, aucun dallage.

L'égout pluvial a un développement modeste puisqu'il est constitué que par deux tronçons, respectivement de $114 \mathrm{~m}$ et de $245 \mathrm{~m}$; il est dépourvu de branchements secondaires; les pentes des tronçons sont très semblables à celles des rues (fig. 2). En outre, les canaux, en béton, ont un diamètre de 250 et $300 \mathrm{~mm}$, avec un volume total à section complètement pleine de $20 \mathrm{~m}^{3}$ environ; les bouches sur chaussée, de forme rectangulaire, ont les dimensions suivantes : $0,50 \mathrm{~m}$ environ par $1.50 \mathrm{~m}$, et occupent presque toujours la largeur de la chaussée.

Cependant, les grilles métalliques, réalisées en rond à béton de $20 \mathrm{~mm} \phi$ avec un écartement de l'ordre de $10 \mathrm{~mm}$, ne facilitent pas la récupération des eaux puisqu'elles sont souvent partiellement obstruées. La majeure partie de l'écoulement se produit donc sur la chaussée où l'eau est retenue pendant quelque temps ou bien elle suit des parcours sinueux avant de rejoindre un des collecteurs. Ceux-ci, enfin, confluent dans un canal à ciel ouvert où se trouve l'appareil de mesure des débits.

\section{Description de la station de mesure}

La station de mesure des précipitations et des écoulements est placée immédiatement à l'aval de l'exutoire du bassin (fig. 1).

Pour l'enregistrement des précipitations on a employé un pluviographe à augets basculeurs, dont le pas correspond à $0.2 \mathrm{~mm}$ de pluie; la rotation du tambour, de $12 \mathrm{~mm} / \mathrm{h}$, actionné par une horloge mécanique, permet d'évaluer la hauteur de pluie tombée dans un intervalle de $5 \mathrm{~min}$.

Pour la mesure des écoulements, on a utilisé un canal à ciel ouvert, dans lequel débouche le collecteur final de la canalisation pluviale, précédé et suivi par deux chutes. Il a $13 \mathrm{~m}$ de long, une section rectangulaire de $0.60 \mathrm{~m}$ de large et de $0.90 \mathrm{~m}$ de haut, et a été recouvert par des grilles métalliques mobiles (fig. 3). Une autre grille verticale, placée à son embouchure, empêche l'entrée de matériel solide grossier.

Un canal jaugeur, préfabriqué en tôle, a été mis en place à distance convenable de la chute à l'aval du canal devant lequel on a posé un seuil; pendant toutes les observations effectuées, on a toujours relevé l'ondulation caractéristique du courant à l'aval de la section critique. 
TRONGSON 1

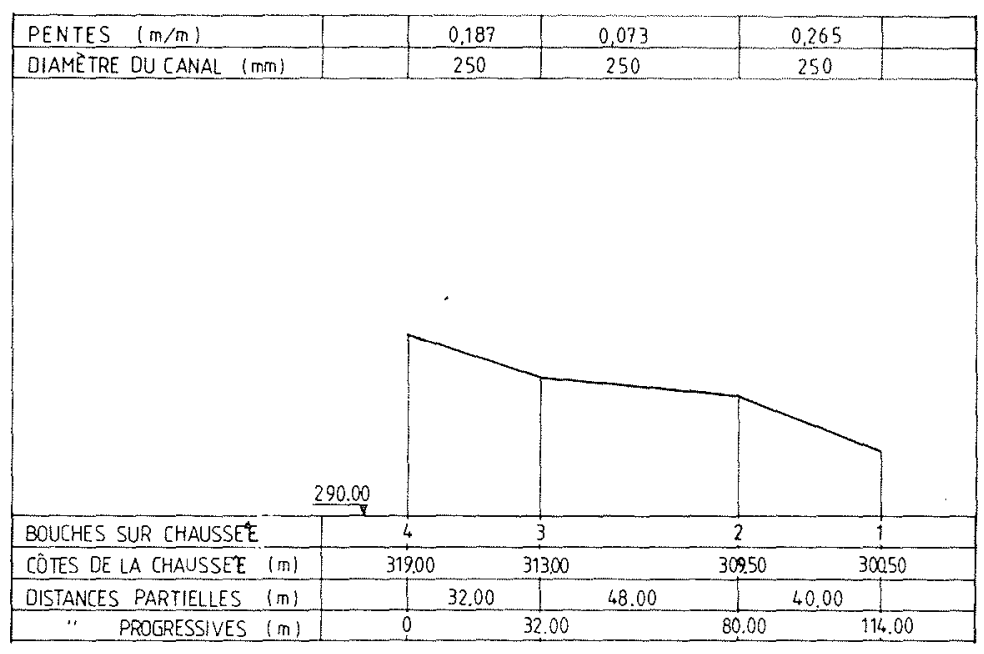

TRONÇON 2

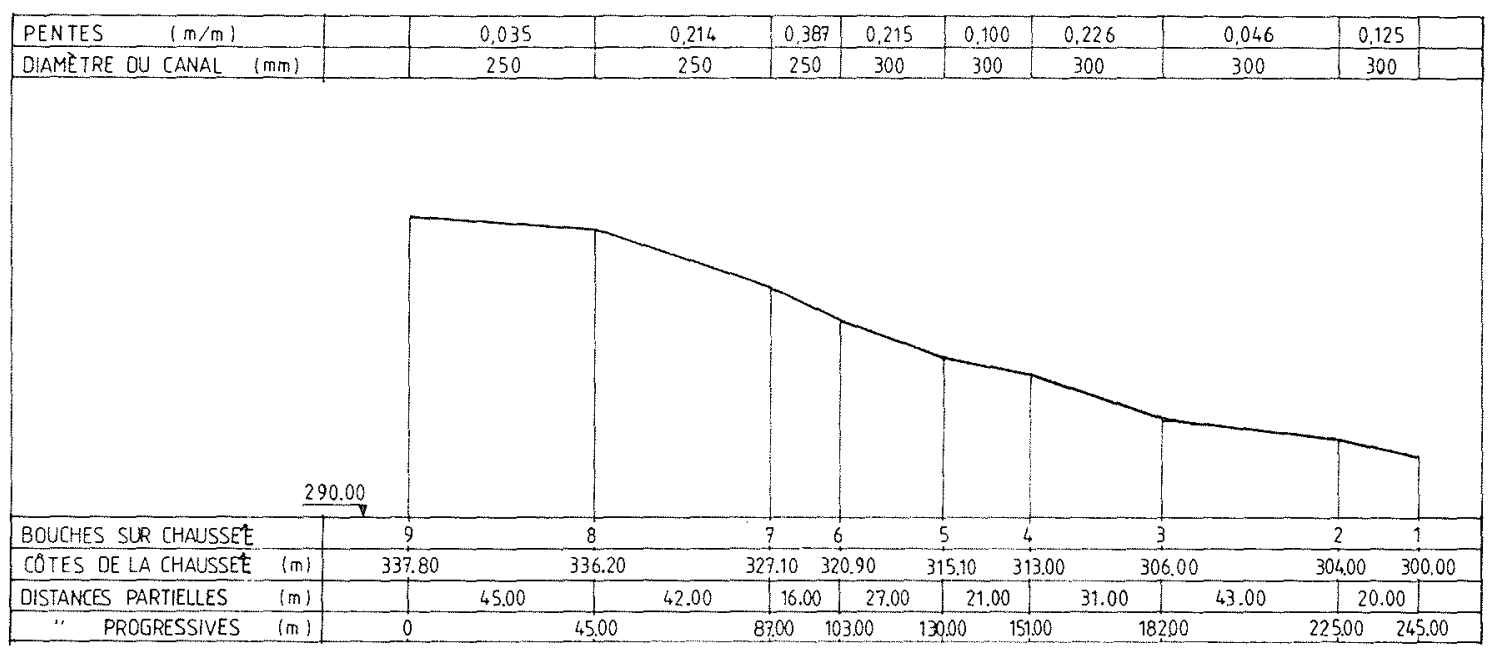

Figure 2 -- Profil en long de l'égout pluvial

L'enregistreur de niveau à flotteur employé a été réglé pour une rotation du tambour de $12 \mathrm{~mm} / \mathrm{h}$, actionné par une horloge mécanique, synchronisée quotidiennement avec celle du pluviographe. On a considéré indispensable, après trois années, de perfectionner l'acquisition des données avec un système basé sur le synchronisme des enregistrements.

\section{Modèles de Nash et du réservoir linéaire}

Pour de petites aires urbanisées les modèles globaux linéaires peuvent simuler de façon satisfaisante la transformation précipitations-écoulements. En fait, ils ne tiennent pas compte de la distribution spatiale de la pluie ni de la variation spatiale des paramètres qui caractérisent la phénomène physique.

Un système linéaire, comme on sait, peut être identifié en assimilant sa réponse $\sqrt{h(t)}$ à une impulsion unitaire $\delta(t)$. Dans ce cas la relation (1) devient :

$$
Q(t)=\int_{0}^{t} I(t-\tau) h(\tau) d \tau
$$

Pour la détermination de l'IUH on a choisi parmi les nombreux modèles conceptuels, ceux du réservoir unique et des $n$ réservoirs linéaires ou de Nash, qui, comme on l'affirme en [16], [17], donnent les meilleurs résultats pour les bassins urbains. L'application de tels modèles semble préférable quand l'effet de capacité est prédominant par rapport aux effets de translation.

Des hyétogrammes enregistrés on a tiré les hyétogrammes efficaces par l'intermédiaire des coefficients d'écoulement observés pour chaque événement. Les hyétogrammes efficaces et les hydrogrammes observés ont permis l'identification des paramètres des deux modèles par la méthode des moments.

Les valeurs des paramètres obtenus pour dix événements observés sont reproduites dans le tableau $\mathrm{I}$, avec 

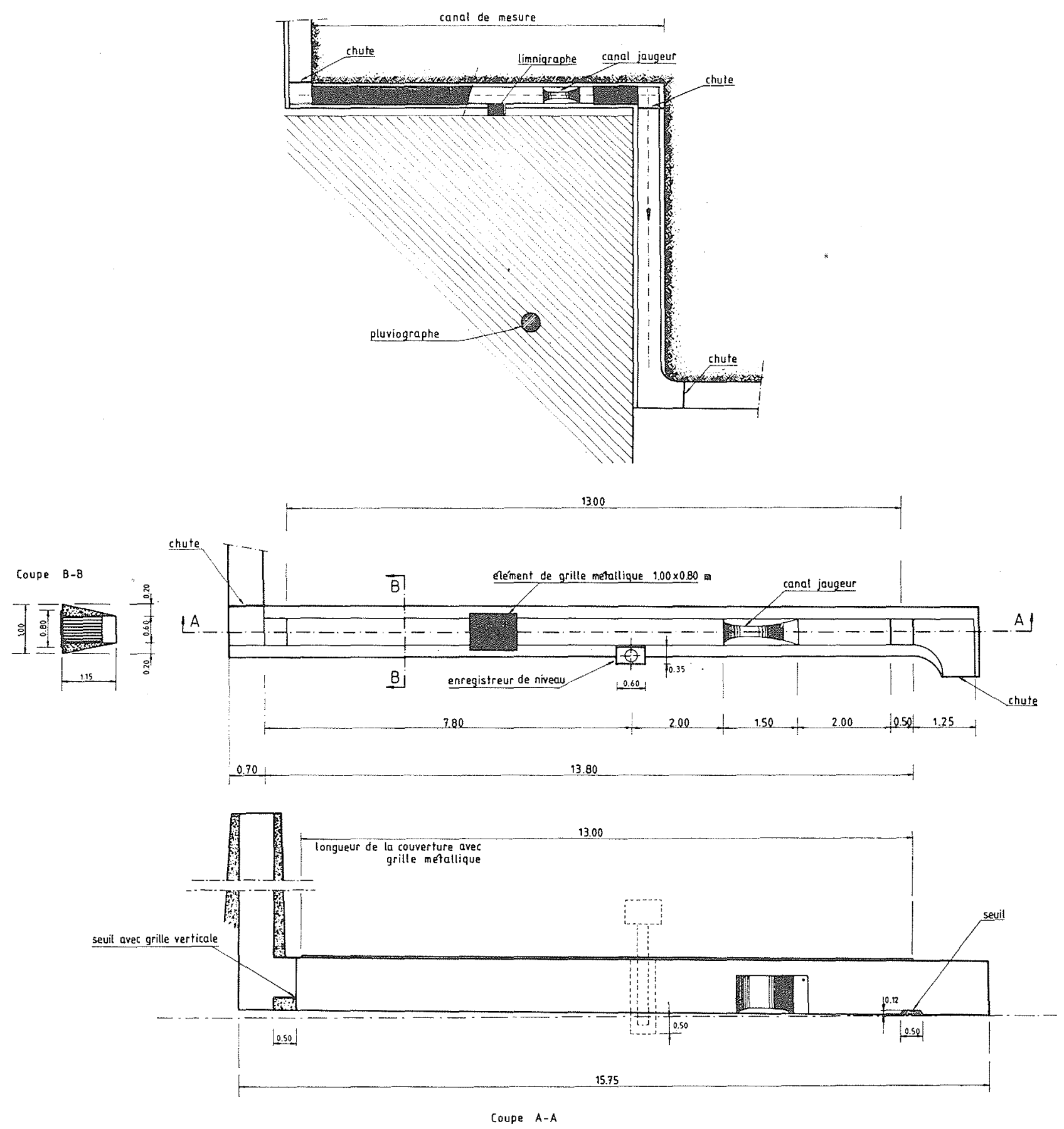

Figure 3 - Station de mesure des précipitations et des écoulements

les valeurs observées du coefficient de ruissellement. Les IUH sont :

$$
h(t)=\frac{1}{k} e^{-t / k}
$$

pour le modèle linéaire du réservo ir, et

$$
h(t)=\frac{1}{k} \frac{e^{-t / k}}{\Gamma(n)}(t / k)^{n-1}
$$

pour le modèle de Nash; les hydrogrammes ont été reconstruits par une intégration discrète de l'intégral de convolution.
L'étude expérimentale a montré que les paramètres $k$ et $n$ ne peuvent pas être considérés comme des invariants du bassin versant. Leurs valeurs dépendent en particulier des antécédents pluviométriques qui jouent sur la valeur du coefficient de ruissellement.

On remarque aussi que la moitié des valeurs du paramètre $k$, dans le modèle du réservoir linéaire, se situe entre 5.52 et 5.80 .

La figure 4 met en évidence la variabilité des noyaux : dans deux cas seulement (événements $n^{\circ} 3$ et $n^{\circ} 9$ ) la pointe initiale de l'IUH est très haute.

Même les valeurs des paramètres $k$ et $n$ du modèle de Nash varient d'un événement à l'autre; celà confirme 


\begin{tabular}{|c|c|c|c|c|c|c|}
\hline \multicolumn{7}{|c|}{ Tableau I } \\
\hline \multirow[b]{2}{*}{$N^{0}$} & \multirow[b]{2}{*}{ jour } & \multirow[b]{2}{*}{ heure } & \multirow[b]{2}{*}{$\phi$} & \multirow{2}{*}{$\begin{array}{c}\text { réserv. unique } \\
k \\
(\mathrm{~min})\end{array}$} & \multicolumn{2}{|c|}{ modèle de Nash } \\
\hline & & & & & $n$ & $(\min )$ \\
\hline 1 & $26 / 06 / 79$ & 23.15 & 0.41 & 5.72 & 1.12 & 5.09 \\
\hline 2 & $12 / 12 / 79$ & 10.30 & 0.51 & 5.52 & 0.54 & 10.26 \\
\hline 3 & $16 / 12 / 79$ & 11.30 & 0.51 & 2.50 & 0.63 & 3.96 \\
\hline 4 & $16 / 12 / 79$ & 12.35 & 0.69 & 7.20 & 2.52 & 2.85 \\
\hline 5 & $23 / 12 / 79$ & 17.30 & 0.41 & 5.53 & 0.55 & 10.00 \\
\hline 6 & $31 / 12 / 79$ & 10.30 & 0.61 & 5.77 & 1.66 & 3.48 \\
\hline 7 & $31 / 12 / 79$ & 16.20 & 0.74 & 5.80 & 0.72 & 8.02 \\
\hline 8 & $31 / 12 / 79$ & 19.15 & 0.88 & 8.04 & 1.54 & 5.20 \\
\hline 9 & $8 / 03 / 80$ & 6.00 & 0.43 & 3.37 & 0.44 & 7.66 \\
\hline 10 & $15 / 03 / 80$ & 11.45 & & & & \\
\hline
\end{tabular}

ce qui a été relevé par d'autres auteurs [17], [18]. La figure 4 met également en évidence la variété des formes des noyaux dans le cas du modèle de Nash. La figure 5 montre la reconstitution des hydrogrammes des événements $n^{\circ} 6,7$ et 10 du tableau 1 .

Les résultats obtenus, certainement insuffisants pour formuler un jugement définitif sur les modèles proposés, encouragent à continuer la recherche, en particulier en vue d'analyser l'incidence des caractéristiques des pluies et des bassins sur la nature des noyaux des modèles. En effet, on a constaté qu'en raison de la nonlinéarité des phénomènes "précipitations-écoulements" les paramètres de l'IUH dépendent de la pluie efficace. Cela peut donc conduire à chercher des relations entre ces paramètres et les caractéristiques de la pluie. Il est pourtant possible d'envisager aussi des effets de nonlinéarité à l'intérieur d'un même événement : on peut ainsi modifier l'intégrale de convolution selon [18] :

$$
Q(t)=\int_{0}^{t} I(t-\tau) h[I(t-\tau), \tau] d \tau
$$

\section{Modèle cinématique}

Dans le modèle cinématique on a, comme on sait [19]

$$
h(t)=\frac{d A(t)}{d t}
$$

où $A(t)$ est la courbe aire-temps du bassin.

Le modèle a été appliqué en évaluant, par une intégration discrète, $Q(t)$ selon la relation (11).

On a fixé des intervalles de temps successifs $O-t_{1}$, $t_{1}-t_{2} \ldots t_{i-1}-t_{i} \ldots t_{n-1}-t_{n}$, où les intensités de pluie efficace sont $i_{1}, i_{2}, \ldots i_{i} \ldots i_{n}$; on a attribué
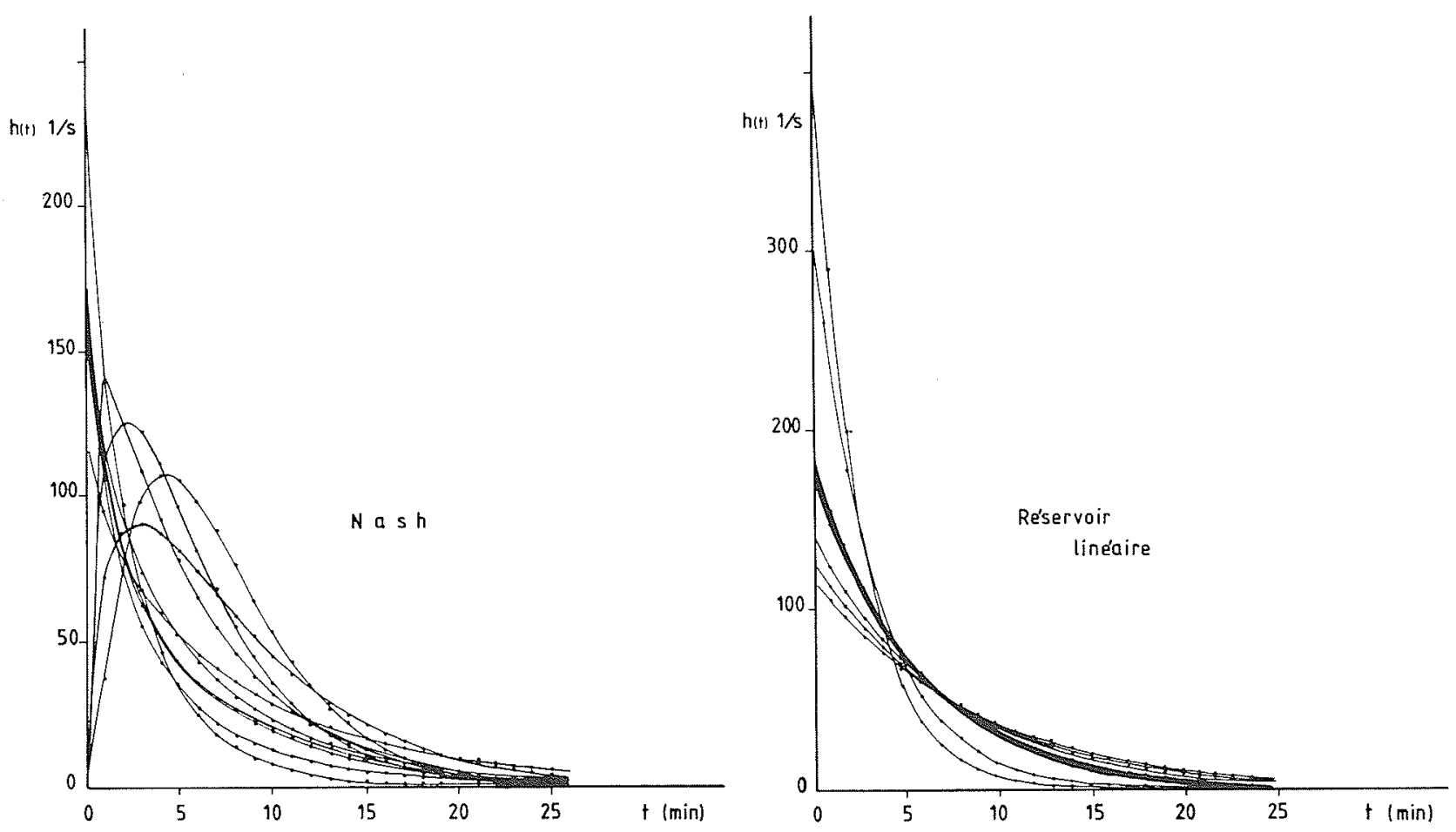

Figure 4 - Noyaux obtenus pour le modèle de Nash et du Réservoir linéaire 

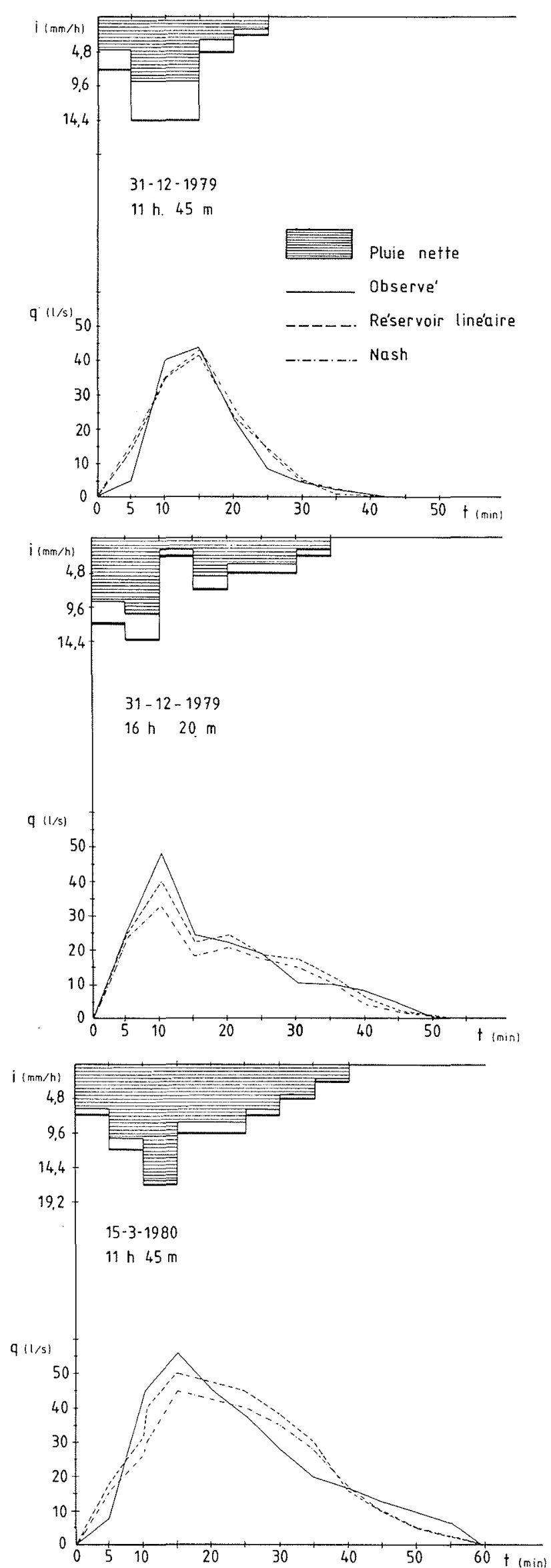

Figure 5 - Hydrogrammes reconstruits suivant le modèle de Nash et du réservoir linéaire à chacun de ces intervalles des $h(t)$ différents, soit $h_{1}(t), h_{2}(t) \ldots h_{i}(t) \ldots h_{n}(t)$.

Le débit au temps $t$, compris entre $t_{i-1}$ et $t_{i}$, est donné par l'équation

$$
\begin{aligned}
Q(t)= & \int_{0}^{t_{1}} i_{1}(t-\tau) h_{1}(\tau) d \tau+ \\
& \int_{t_{1}}^{t_{2}} i_{2}(t-\tau) h_{2}(\tau) d \tau+ \\
& \int_{t_{i-1}}^{t} i_{i}(t-\tau) h_{i}(\tau) d \tau
\end{aligned}
$$

Pour des petits bassins urbains, il est conseillé [20], [21] de considérer $A(t)$ linéaire; dans ce cas, $h(t)$ a une valeur constante à l'intérieur de chaque intervalle de temps, soit

$$
h(t)=\frac{A_{T}}{t_{c}}
$$

où $A_{T}$ est l'aire totale du bassin, et $t_{c}$ le temps de concentration relatif au même intervalle. L'évaluation des paramètres a été conduite par une prise en compte des caractéristiques physiques du bassin.

En effet, pour l'évaluation de $t_{c}$ on peut écrire

$$
t_{c}=t_{r}+t_{\dot{e}}
$$

où $t_{r}$ est le temps de ruissellement et $t_{\dot{e}}$ le temps d'écoulement dans la canalisation.

Pour $t_{r}(\mathrm{~min})$ on peut écrire [22]

$$
t_{r}=\frac{6.94\left(L_{r}, n\right)^{0.6}}{i^{0.4} s^{0.3}}
$$

où $L_{y}$ est la longueur maximale de l'écoulement superficiel $(m)$

$n$ le coefficient de rugosité de Manning $\left(m^{-1 / 3} s\right)$ $i$ l'intensité de pluie $(\mathrm{mm} / \mathrm{h})$

$s$ la pente moyenne.

Le temps $t_{\dot{e}}$ peut être évalué en supposant, pour des collecteurs de longueur limitée $L_{\dot{e}}$, une vitesse constante $v_{e}$.

Pour chaque intervalle de temps assez petit on peut enfin supposer une valeur $i(t)$ constante, ce qui simplifie les calculs relatifs à la relation (12)

Pour l'estimation des intensités de pluie efficace, on a proposé d'adopter un coefficient de ruissellement $\phi(t, b)$, fonction non seulement du temps évalué dès le début de la précipitation, mais aussi de la valeur de l'intensité $i(t)$ et des conditions in itiales du bassin, exprimées par une constante $b$ pour toute la durée de l'évènement.

On peut donc écrire :

$$
\phi(t, b)=\frac{i(t) t}{i(t) t+b}
$$

Cette constante $b$ peut être calculée en observant que, pour l'égalité des volumes écoulés, on doit obtenir :

$$
\phi \Sigma i(t)=\Sigma \frac{i^{2}(t) t}{i(t) t+b}
$$

où $\phi$ est le coefficient de ruissellement relatif à l'évènement total. 
Le modèle décrit a été appliqué en découpant le bassin expérimental en deux sous-bassins qui confluent au même exutoire (Fig. 1); leurs caractéristiques sont données dans le tableau II.

\begin{tabular}{|c|c|c|c|c|c|}
\hline \multicolumn{7}{|c|}{ Tableau II } \\
\hline $\begin{array}{c}\text { Sous- } \\
\text { bassin } \\
\mathrm{n}^{\circ}\end{array}$ & $\begin{array}{c}A \\
\left(\mathrm{~m}^{2}\right)\end{array}$ & $\begin{array}{c}L_{r} \\
(\mathrm{~m})\end{array}$ & $s$ & $\begin{array}{c}n \\
\left(\mathrm{~m}^{-1 / 3} s\right)\end{array}$ & $\begin{array}{c}L_{\dot{e}} \\
(\mathrm{~m})\end{array}$ \\
\hline 1 & 7370 & 135 & 0.25 & 0.02 & 114 \\
2 & 9970 & 90 & 0.15 & 0.02 & 245 \\
\hline
\end{tabular}

Les hyétogrammes ont été subdivisés en hyétogrammes élémentaires d'une durée de $5 \mathrm{~min}$, à chacun desquels on a attribué une intensité constante de précipitation:

En fixant $v_{\dot{e}}=1.2 \mathrm{~m} / \mathrm{s}$ on a obtenu les valeurs de $t_{\dot{e}}$, tandis que les valeurs de $t_{r}$ ont été déduites de la relation (14) avec $n=0.02$. Pour chaque intervalle de $5 \mathrm{~min}$ on a ensuite évalué $h(t)$ par la relation (13).

La pluie efficace a été déduite en calculant par (16) la constante $b$ pour chaque événement et les $\phi(t, b)$ successifs par l'équation (15). Les hydrogrammes relatifs à chacun des deux sous-bassins ont été reconstruits par (12), et l'hydrogramme global a été obtenu en superposant les deux hydrogrammes.

Les résultats obtenus pour dix événements observés sont donnés dans le tableau III.

Les résultats de la reconstruction des hydrogrammes relatifs aux événements $n^{\circ} 6,7$ et 10 sont reproduits sur la figure 6 . On a pas remarqué de différences sensibles en faisant varier la vitesse $V_{\dot{e}}$ et le coefficient $n$ de Manning dans l'équation (14).

Une étude systématique et générale de la sensibilité du modèle permettra de définir la précision avec laquelle on doît connaitre les paramètres du modèle pour une définition correcte de l'hydrogramme résultant d'une entrée donnée.

\section{Hyétogramme de projet}

Afin de calculer le débit de projet, il faut non seulement trouver le modèle le plus adapté à la simulation du processus de transformation précipitations-écoule- ments, mais aussi déterminer la précipitation synthétique, qui tienne compte des propriétés stochastiques des événements observés.

On préfère recourir à l'emploi d'un hyétogramme de projet, plutôt qu'à une précipitation d'intensité constante et égale à la valeur moyenne correspondante à la durée de l'évènement. En effet, si l'on veut tenir compte du fait que des pluies ayant la même durée et la même intensité moyenne, peuvent donner lieu à des hydrogrammes différents, soit dans leur forme, soit dans leur valeur de la pointe, on ne peut pas faire abstraction de la variation de l'intensité pendant l'évènement considéré.

Si l'on utilise la courbe intensité-durée fréquence, même en modifiant la position de l'intensité maximale, on parvient à un hyétogramme de probabilité inconnue.

Il s'agit, par contre, de déterminer un hyétogramme dérivé de l'analyse de tous les orages enregistrés, c'est-àdire un hyétogramme synthétique de projet, qui soit en accord avec les propriétés stochastiques relatives à la variation des intensités de pluie pendant l'évènement. Ces propriétés sont le volume total d'eau tombée, la location temporelle de l'intensité maximale et le volume de pluie tombée avant celle-ci. La méthode conseillée en [1] tient compte des volumes de pluie tombés avant l'intensité maximale. Sur la figure $7 \mathrm{a}$ on a reproduit le résultat d'une telle application, relative aux orages de tableau IV.

\begin{tabular}{|r|r|r|r|r|}
\hline \multicolumn{5}{|c|}{ Tableau IV } \\
\hline$N^{\circ}$ & jour & heure & $\begin{array}{c}\text { durée } \\
(\mathrm{min})\end{array}$ & $\begin{array}{c}\text { intensité max } \\
(\mathrm{mm} / \mathrm{h})\end{array}$ \\
\hline 1 & $20 / 03 / 79$ & 14.10 & 20 & 14.10 \\
2 & $20 / 06 / 79$ & 23.15 & 20 & 14.40 \\
3 & $21 / 06 / 79$ & 2.35 & 20 & 28.80 \\
4 & $12 / 12 / 79$ & 10.30 & 20 & 28.80 \\
5 & $16 / 12 / 79$ & 11.30 & 15 & 12.00 \\
6 & $16 / 12 / 79$ & 12.35 & 20 & 14.40 \\
7 & $23 / 12 / 79$ & 17.30 & 25 & 12.00 \\
8 & $31 / 12 / 79$ & 10.30 & 25 & 14.40 \\
9 & $31 / 12 / 79$ & 16.20 & 35 & 14.40 \\
10 & $31 / 12 / 79$ & 19.15 & 20 & 12.00 \\
11 & $8 / 03 / 80$ & 6.00 & 20 & 12.00 \\
12 & $15 / 03 / 80$ & 11.45 & 40 & 16.80 \\
13 & $13 / 10 / 80$ & 16.25 & 15 & 21.60 \\
14 & $14 / 10 / 80$ & 0.50 & 15 & 16.80 \\
\hline
\end{tabular}

\begin{tabular}{|c|c|c|c|c|c|c|}
\hline \multicolumn{7}{|c|}{ Tableau III } \\
\hline$N^{\circ}$ & jour & heure & \multicolumn{2}{|c|}{$\phi \underset{(\mathrm{mm} \mathrm{min} / \mathrm{h})}{ } b$} & \multicolumn{2}{|c|}{$\begin{array}{c}q_{\text {pointe }} \\
(\ell / s) \\
\text { observée calculée }\end{array}$} \\
\hline 1 & $26 / 06 / 79$ & 23.15 & 0.41 & 160.0 & 40 & 31 \\
\hline 2 & $12 / 12 / 79$ & 10.30 & 0.51 & 135.0 & 48 & 52 \\
\hline 3 & $16 / 12 / 79$ & 11.30 & 0.51 & 52.0 & 35 & 20 \\
\hline 4 & $16 / 12 / 79$ & 12.35 & 0.69 & 27.5 & 38 & 32 \\
\hline 5 & $23 / 12 / 79$ & 17.30 & 0.41 & 157.0 & 25 & 24 \\
\hline 6 & $31 / 12 / 79$ & 10.30 & 0.61 & 72.8 & 42 & 50 \\
\hline 7 & $31 / 12 / 79$ & 16.20 & 0.74 & 34.0 & 48 & 48 \\
\hline 8 & $31 / 12 / 79$ & 19.15 & 0.88 & 8.5 & 35 & 48 \\
\hline 9 & $8 / 03 / 80$ & 6.00 & 0.43 & 108.5 & 26 & 20 \\
\hline 10 & $15 / 03 / 80$ & 11.45 & 0.87 & 20.8 & 56 & 68 \\
\hline
\end{tabular}



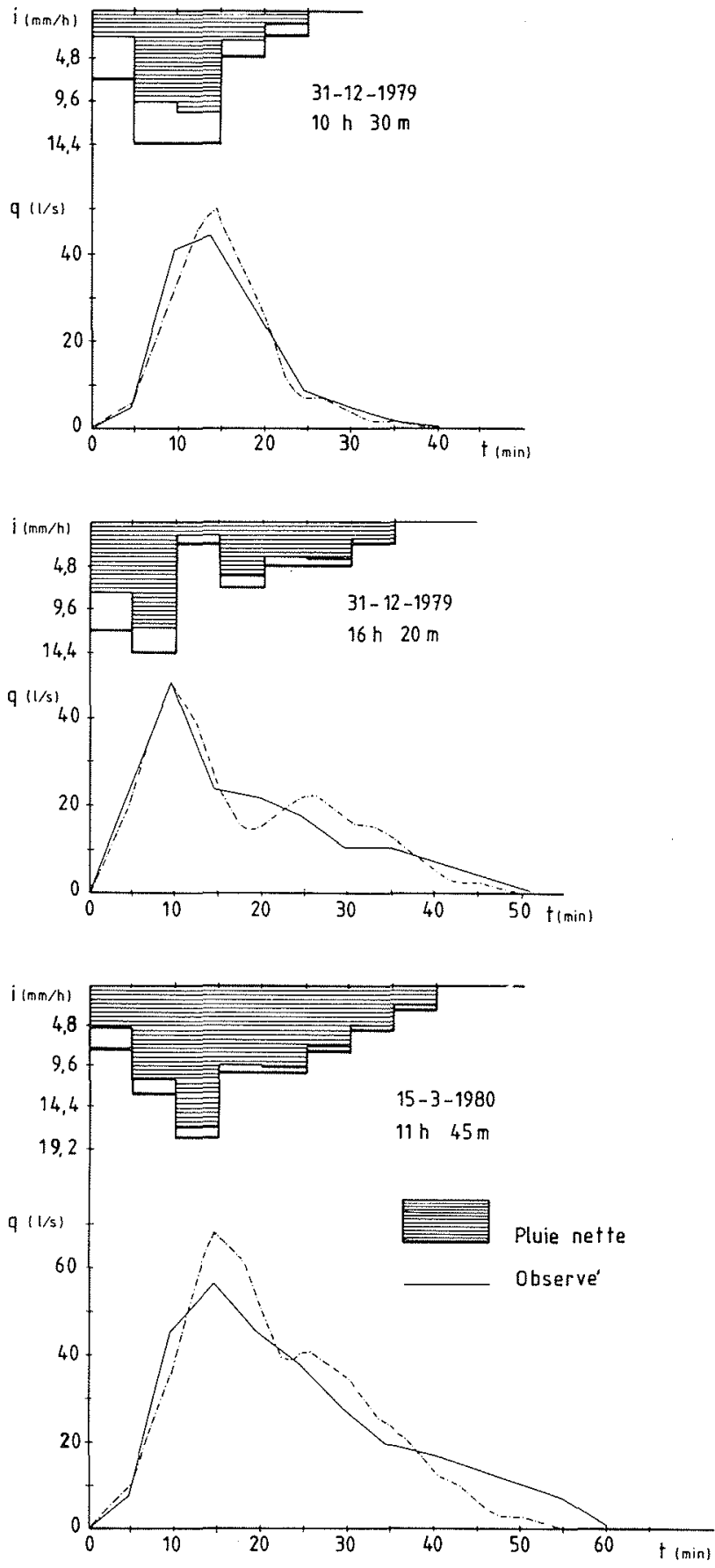

Figure 6 - Hydrogrammes reconstruits selon la méthode cinématique

On peut parvenir à la détermination du volume de la pluie antécédente aussi par évaluation statistique de la position du maximum suivant la méthode proposée par [23], et reprise par d'autres. Sur la figure $7 b$ on trouve le résultat de cette application.

Un défaut de ces méthodes, qui constituent pourtant un progrès par rapport à l'emploi de la courbe "intensitédurée", réside dans le fait qu'il faut disposer d'un grand nombre d'enregistrement d'orages. Il semble donc utile, dans le cas où l'on ne peut disposer d'un certain nombre de données enregistrées, de se servir d'un des nombreux modèles stochastiques pour engendrer des séries synthétiques. Parmi ceuxtà, la méthode de la matrice de la
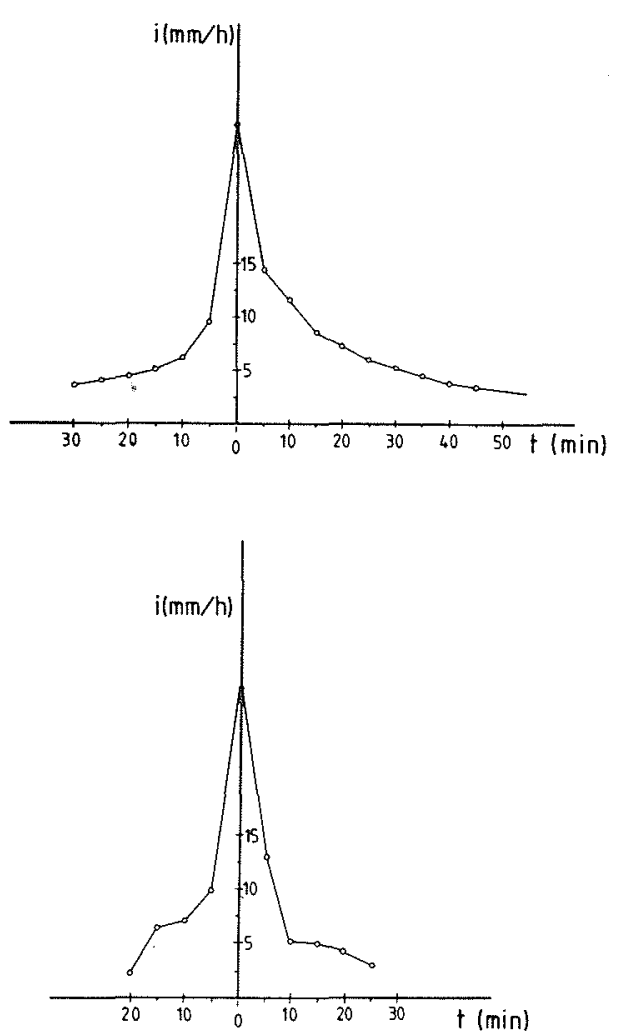

Figure 7 - Pluviogrammes de projet

probabilité de transition pourrait être valable, du moment que, permettant la génération de séquences synthétiques de pluie de courte durée, elle peut être employée pour la génération d'orages synthétiques.

Il est pourtant vrai que l'analyse, statistique, si l'on dispose d'un nombre suffisant de données, peut être limitée à quelques paramètres qui définissent la pluie de projet, comme l'intensité moyenne sur durées typiques et la position de la pointe [26].

La détermination de l'hyétogramme de projet est indispensable quand on veut parvenir à la simulation d'un hydrogramme de crue avec n'importe quel modèle mathématique connu. Quand, dans des cas particuliers, il peut être utile de recourir à la méthode rationnelle, très employée dans la pratique, il n'est pas strictement nécessaire de déterminer l'hyétogramme de projet, puisque la méthode fournit la valeur de la pointe et non l'hydrogramme de crue. Cependant, même quand on a l'intention d'appliquer cette méthode, la connaissance de l'hyétogramme de projet est indispensable pour déterminer la valeur exacte du coefficient de ruissellement.

\section{Conclusions}

La qualité et la non-exceptionnalité des événements enregistrés ne permettent pas de déduire des résultats définitifs à partir des tests effectués.

Des enregistrements plus nombreux de pluie et de débit permettront d'optimiser les paramètres des différents modèles adoptés. Il sera donc possible de faire une comparaison entre leurs performances et celles 
d'autres modèles que l'on estimera opportun d'appliquer au bassin à l'étude.

Afin d'acquérir des mesures plus précises, le bassin vient d'être équipé grâce à la contribution du C.N.R: d'une station automatique assurant le synchronisme parfait des enregistreurs et un pas de temps de lecture de l'ordre de la minute, mieux adapté aux caractéristiques des événements observés.

\section{Références}

[1] Commissione per lo studio delle portate di piena delle fognature. - Relazione. Consiglio Nazionale delle Ricerche, Roma.

[2] ALLISON S.V. - Review of small basin Runoff prediction methods. Joumal of the Irrigation and Drainage Division, ASCE, Vol. 93, No. IR1, March, 1967, pp. 1-6.

[3] MC CUEN R.H. and RAWLS W.J. - Classification of evaluation of flood flow frequency estimation techniques. Water Resources Bulletin, Vol 15, No. 1, February, 1979 , pp. 88-93.

[4] VEN THE CHOW and KULANDAISWAMY V.C. - General hydrologic system model. Joumal of the Hydraulics Division, $A S C E$, Vol. 97, No. HY6, June, 1971, pp. 791-804.

[5] ROGERS R.A. - Rational "rational" method of storm drainage design. Journal of the Irrigation and Drainage Division, ASCE, Vol. 94, No. IR4, December, 1968, pp. $465-479$.

[6] SCHAAKE J.C., GEYER J.C. and KNAPP J.W. - Experimental examination of the rational method. Journal of the Hydraulics Division, ASCE, Vol. 93, No. HY6, November, 1967, pp. 353-370.

[7] DESBORDES M. - Extension du modèle de Caquot aux bassins versants hétérogènes. Techniques et Sciences Municipales, No. 5, Mai, 1976 , pp. 223-229.

[8] PAOLETTI A., PAPIRI S. - Ricerca sperimentale sulla formazione delle piene in una rete fognante. Primi risultati. Ingegneria Ambientale, Vol. No. 3, Maggio-Giugno, 1980 , pp. 187-198.

[9] DESBORDES M. - Application des, modèles aux études de ruissellement urbain : le programme RERAM. La Houille Blanche, Numéro Spécial, No. 4/5, 1980, pp. 333-337.

[10] CHEN C.W. and SHUBINSKI R.P. - "Computer simulation of urban storm water Runoff. Joumal of the Hydraulics Division, ASCE, Vol. 97, No. HY2, February, 1971, pp. 289-301.

[11] PHAMWON S. and YU-SI FOK. - Urban Runoff digital computer model. Joumal of the Hydraulics Division, ASCE, Vol 103, No. HY7, July, 1977, pp. 723-735.
[12] PAPADAKIS C. and PREUL H.C. - University of Cincinnati urban Runoff model. Journal of the Hydraulics Division, ASCE, Vol. 98, No. HY 10, October, 1972, pp. 17891804.

[13] MODICA C., QUIGNONES R., ROSSI G. - Contributo al calcolo di progetto di reti di fognatura pluviale. Idrotecnica, No. 6, Novembre/Dicembre, 1979, pp. 251-269.

[14] DOOGE J.C.I. - Conceptual models of surface Runoff Atti del convegno Internazionale sul Tema "Piene : loro previsione e difesa del suolo", Quaderno No. 169, Accademia Nazionale dei Lincei, Roma, 1972, pp. 179-207.

[15] DOOGE J.C.I. - Problems and methods of rainfall-Runoff modelling. Workshop on Mathematical Models in Hydrology, Pisa, December, 1974, pp. 71-108.

[16] RAO R.A., DELLEUR J.W. and SARMA B.S.P. - Conceptual hydrologic models for urbanizing basins. Journal of the Hydraulics Division, ASCE, Vol. 98, No. HY7, July, 1972, pp. 1205-1220.

[17] SARMA B.S.P., DELLEUR J.W. and RAO A.R. - Comparison of rainfall-Runoff models for urban areas. Journal of Hydrology, Vol. XVIII, 1973, pp. 329-347.

[18] DING J.Y. - Variable unit hydrograph. Jurnal of Hydrology, Vol. 22, 1974, pp. 53-69.

[19] MAIONE U. - Appunti di Idrologia, Vol. 3, Le piene fluviali, La Goliardica Pavese, 1977.

[20] JONG-SONG-CHIEN and SAIGAL K.K. - Urban Runoff by linearized subhydrographic method. Joumal of the Hydraulics Division, ASCE, Vol. 100, No. HY8, August, 1974, pp. 1141-1157.

[21] WALESH S.G. - Urban Runoff by linearised subhydrographic method, discussion. Journal of the Hydraulics Division, ASCE, Vol. 101, No. HY11, November, 1975, pp. 1447-1449.

[22] RAGAN R.M. and DURU J.O. - Kinematic wave nomograph for times of concentration. Journal of the Hydratulics Division, $A S C E$, Vol. 98, No. HY10, October, 1972, pp. $1765-1771$.

[23] KEIFER C.J. and CHU H.H. - Synthetic storm pattern for drainage design. Journal of the Hydraulics Division, ASCE, Vol. 83, No. HY4, August, 1957, pp. 1332-1$1332-25$.

[24] PREUL H.C. and PAPADAKIS C.N. - Development of design storm hyetographs for Cincinnati, Ohio. Water Resources Bulletin, Vol. 9, No. 2, April, 1973, pp. 291300.

[25] BANDYOPADHYAY M. - Synthetic storm pattern and run-off for Gauhati, India. Journal of the Hydraulics Division, ASCE, Vol. 98, No. HY5, May, 1972, pp. 845857.

[26] DESBORDES M. et RAOUS P. - Un exemple de l'intérêt des études de sensibilité des modèles hydrologiques. $L a$ Houille Blanche, No. 1, 1976, pp. 37-43. 